\title{
On the Stability of $L_{4,5}$ in the Relativistic R3BP with Oblate Secondary and Radiating Primary
}

\author{
Nakone Bello ${ }^{1}$ and Jagadish Singh ${ }^{2}$ \\ ${ }^{1}$ Department of Mathematics, Faculty of Science, Usmanu Danfodiyo University, PMB 2346, Sokoto, Nigeria \\ ${ }^{2}$ Department of Mathematics, Faculty of Science, Ahmadu Bello University, PMB 1044, Zaria, Kaduna State, Nigeria \\ Correspondence should be addressed to Nakone Bello; bnakone@yahoo.com
}

Received 8 April 2015; Revised 17 June 2015; Accepted 18 June 2015

Academic Editor: Elmetwally Elabbasy

Copyright (c) 2015 N. Bello and J. Singh. This is an open access article distributed under the Creative Commons Attribution License, which permits unrestricted use, distribution, and reproduction in any medium, provided the original work is properly cited.

\begin{abstract}
We consider a version of the relativistic restricted three-body problem (R3BP) which includes the effects of oblateness of the secondary and radiation of the primary. We determine the positions and analyze the stability of the triangular points. We find that these positions are affected by relativistic, oblateness, and radiation factors. It is also seen that both oblateness of the secondary and radiation of the primary reduce the size of stability region. Further, a numerical exploration computing the positions of the triangular points and the critical mass ratio of some binaries systems consisting of the Sun and its planets is given in the tables.
\end{abstract}

\section{Introduction}

The problem of three bodies moving under their mutual gravitation attraction has been a subject of keen interest to interested mathematicians since Newton [1] who first postulated the system as three bodies whose forces decrease in a duplicate ratio of the distances, attracting each other mutually; and the accelerative attractions of any two towards the third are between themselves reciprocally as the squares of the distances. Poincare [2] proved that no analytic integrals of motion exist for the general three-body problem other than the energy and angular momentum. This implies that the problem may not be solved in terms of algebraic functions and integrals, but only through numerical integration or infinite series representation.

Regarding the general three-body problem, we may cite Chenciner [3] and Valtonen and Karttunen [4]. There are also various forms of three-body problems in general relativity, for which we may cite Renzetti [5], Nordtvedt [6], and Iorio [7]. If the mass of any one of the three bodies is negligible as compared to the mass of the other two bodies, then it imposes no gravitational influence on the motion of the other two. This then introduces a special form of the three-body problem referred to as the restricted three-body problem (R3BP). The larger bodies are referred to as the primaries and the third body as spacecraft or test particle.
In 1765, Euler [8] found a collinear solution for the restricted three-body problem, where one of the three bodies is a test mass. Soon later, his solutions were extended for a general three-body problem by Lagrange [9] who also found an equilateral triangular solutions in 1772 . Now, the solutions for the restricted three-body problem are called Lagrange points $L_{1}, L_{2}, L_{3}, L_{4}$, and $L_{5}$. The collinear points $L_{1}, L_{2}$, and $L_{3}$ are unstable, they lie on the line joining the primaries, and the triangular points $L_{4}$ and $L_{5}$ are stable for the mass ratio $\mu<0.03852 \ldots[10]$.

The solar and heliospheric observations (SOHO) and WMAP launched by NASA are in operation at $L_{1}$ and $L_{2}$ of the Sun-Earth system, respectively. The laser interferometer space antenna (LISA) pathfinder is planned to go to $L_{1}$. Lagrange points have recently attracted renewed interests for relativistic astrophysics [11, 12], where they have discussed gravitational radiation reaction on $L_{4}$ and $L_{5}$ by numerical methods. As a pioneering work, Nordtvedt [6] pointed out that the location of the triangular points is very sensitive to the ratio of the gravitational mass to the inertial one.

The Lagrangian points, by considering one or both primaries are oblate spheroids, whose equatorial planes coincide with the plane of motion are discussed by Subbarao and Sharma [13] and Markellos et al. [14]. Subbarao and Sharma [13] studied the problem in a synodic coordinates system 
when the massive primary is an oblate spheroid and found that the range of stability decreases with the increase of oblateness factor.

As we know stars (including the Sun) have not only gravitational interaction but also radiation pressure on celestial bodies moving around them. These two actions can be expressed as an equivalent force, called a photogravitation. It is practical and important to study the motion of a small body under the photogravitational restricted three-body problem. Radzievskii $[15,16]$ formulated the photogravitational restricted three-body problem. Sharma [17] studied the linear stability of triangular libration points of the restricted threebody problem when the more massive primary is a source of radiation and oblate spheroid as well. He found that the eccentricity of the conditional retrograde elliptic periodic orbits around the triangular points at the critical mass $\mu_{c}$ increases with the increase in the oblateness coefficient and the radiation force and becomes unity when $\mu_{c}$ is zero.

Simmons et al. [18] gave a complete solution of the restricted three-body. They discussed the existence and linear stability of the equilibrium points for all values of radiation pressures of both luminous bodies and all values of mass ratios.

Sharma [19] studied the stationary solution of the planner restricted three-body problem when the smaller primary is an oblate spheroid with its equatorial plane coinciding with the plane of motion and the bigger primary is a source of radiation as well. He found that the collinear points have conditional retrograde elliptical periodic orbits around them in the linear sense, while the triangular points have long or short periodic retrograde elliptic orbits when parameter of mass is in the range $0 \leq \mu<\mu_{\text {crit }}$, and he deduced that the value of critical mass decreases with the increase of oblateness and radiation force.

AbdulRaheem and Singh [20] studied the stability of equilibrium points under the influence of small perturbations in the Coriolis and the centrifugal forces, together with the effects of oblateness and radiation pressures of primaries, and they found that the Coriolis force has a stabilizing tendency, while the centrifugal force, radiation, and oblateness have destabilizing effects; therefore, the overall effect is that the range of stability of the triangular point decreases.

The existence of libration points and their linear stability as well as periodic orbits around these points when the more massive primary is radiating and smaller is an oblate spheroid studied by Abouelmagd and Sharaf [21]. Their study includes the effects of oblateness of $\bar{J}_{2 i}(i=1,2)$ with respect to the smaller primary in the restricted three-body problem.

In contrast to the Newtonian theory of gravitation, the general relativity equations of motion of the gravitating masses as the source of the field are intimately related to the field equation as was demonstrated by Einstein and Grommer [22]. This is due to the nonlinear form of the field equations and the existence of the four Bianchi identities.

In general relativity, even writing down the equations of motion in the simplest case $N=2$ is difficult. Unlike in
Newton's theory, it is impossible to express the acceleration by means of the positions and velocities, in a way which would be valid within the "Exact" theory. Therefore, the approximation method is needed.

Historically, the equations of motion of the problem of $N$ bodies considered to be point masses were first obtained by generalizing the geodesic principle. By the use of this method de Sitter [23] first derived the relativistic equations of motion of $\mathrm{N}$-body problem. Some arithmetic errors occurred in these equations and are reproduced in the encyclopedic paper of Kottler [24] and the treatise by Chazy $[25,26]$ but were corrected by Eddington and Clark [27]. field.

It is important to highlight some important articles in this

Krefetz [28] computed the post-Newtonian deviations of the triangular Lagrangian points from their classical positions in a fixed frame of reference for the first time, but without explicitly stating the equations of motion.

Brumberg $[29,30]$ studied the problem more in detail and collected most of the important results on relativistic celestial mechanics. He did not obtain only the equations of motion for the general problem of the three bodies but also deduced the equations of motion for the restricted problem of three bodies.

Bhatnagar and Hallan [31] studied the existence and stability of the triangular points $L_{4,5}$ in the relativistic R3BP, and they concluded that $L_{4,5}$ are always unstable in the whole range $0 \leq \mu \leq 1 / 2$ in contrast to the previous results of the classical restricted three-body problem where they are stable for $0 \leq \mu<\mu_{0}$, where $\mu$ is the mass ratio and $\mu_{0}=0.03852 \ldots$ is Routh's value.

Douskos and Perdios [32] examined the stability of the triangular points in the relativistic R3BP and, contrary to the results of Bhatnagar and Hallan [31], they obtained a region of linear stability in the parameter space $0 \leq \mu<\mu_{0}-$ $17 \sqrt{69} / 486 c^{2}$ where $\mu_{0}=0.03852 \ldots$ is Routh's value.

The locations of libration points in the relativistic R3BP when including one or more additional effects are included in the potential due to radiation pressure and oblateness of the primaries was studied by $[33,34]$. The locations of triangular points and their linear stability when the bigger primary is radiating in the relativistic R3BP were examined by Singh and Bello [35]. In all the studies previously mentioned in the relativistic R3BP, no work is performed in the direction of linear stability of triangular points in the presence of both radiation and oblateness.

Our interest in the present paper is not only to obtain the locations of the triangular points but also to study their linear stability when the bigger primary is radiating and smaller one is oblate.

This paper is organized as follows: In Section 2, the equations governing the motion are presented; Section 3 describes the positions of triangular points, while their linear stability is analyzed in Section 4. A discussion of these results and their numerical applications are given in Sections 5 and 6 , respectively; finally Section 7 summarizes the conclusions of our paper. 


\section{Equations of Motion}

The pertinent equations of motion of an infinitesimal mass in the relativistic R3BP in barycentric synodic coordinate system $(\xi, \eta)$ and dimensionless variables can be written as Brumberg [29] and Bhatnagar and Hallan [31]:

$$
\begin{aligned}
& \ddot{\xi}-2 n \dot{\eta}=\frac{\partial W}{\partial \xi}-\frac{d}{d t}\left(\frac{\partial W}{\partial \dot{\xi}}\right), \\
& \ddot{\eta}+2 n \dot{\xi}=\frac{\partial W}{\partial \eta}-\frac{d}{d t}\left(\frac{\partial W}{\partial \dot{\eta}}\right)
\end{aligned}
$$

with

$$
\begin{aligned}
W & =\frac{1}{2}\left(\xi^{2}+\eta^{2}\right)+\frac{1-\mu}{\rho_{1}}+\frac{\mu}{\rho_{2}}+\frac{1}{c^{2}}\left[-\frac{3}{2}(1\right. \\
& \left.-\frac{1}{3} \mu(1-\mu)\right)\left(\xi^{2}+\eta^{2}\right)+\frac{1}{8}\left\{\dot{\xi}^{2}+\dot{\eta}^{2}+2(\xi \dot{\eta}-\eta \dot{\xi})\right. \\
& \left.+\left(\xi^{2}+\eta^{2}\right)\right\}^{2}+\frac{3}{2}\left(\frac{1-\mu}{\rho_{1}}+\frac{\mu}{\rho_{2}}\right)\left(\dot{\xi}^{2}+\dot{\eta}^{2}\right. \\
& \left.+2(\xi \dot{\eta}-\eta \dot{\xi})+\left(\xi^{2}+\eta^{2}\right)\right)-\frac{1}{2}\left(\frac{(1-\mu)^{2}}{\rho_{1}^{2}}+\frac{\mu^{2}}{\rho_{2}^{2}}\right) \\
& +\mu(1-\mu)\left\{\left(4 \dot{\eta}+\frac{7}{2} \xi\right)\left(\frac{1}{\rho_{1}}-\frac{1}{\rho_{2}}\right)\right. \\
& -\frac{\eta^{2}}{2}\left(\frac{\mu}{\rho_{1}^{3}}+\frac{1-\mu}{\rho_{2}^{3}}\right) \\
& \left.\left.+\left(\frac{-1}{\rho_{1} \rho_{2}}+\frac{3 \mu-2}{2 \rho_{1}}+\frac{1-3 \mu}{2 \rho_{2}}\right)\right\}\right], \\
n & =1-\frac{3}{2 c^{2}}\left(1-\frac{1}{3} \mu(1-\mu)\right), \\
\rho_{1}^{2} & =(\xi+\mu)^{2}+\eta^{2}, \\
\rho_{2}^{2} & =(\xi+\mu-1)^{2}+\eta^{2},
\end{aligned}
$$

where $0<\mu \leq 1 / 2$ is the ratio of the mass of the smaller primary to the total mass of the primaries; $\rho_{1}$ and $\rho_{2}$ are distances of the infinitesimal mass from the bigger and smaller primary, respectively; $n$ is the mean motion of the primaries; $c$ is the velocity of light.

We now introduce the oblateness effect of the smaller primary and in the radiation effect of the bigger primary with the help of the parameters $A_{2}\left(0 \leq A_{2} \ll 1\right)$ and $q_{1}\left(0<1-q_{1} \ll 1\right)$, respectively. Ignoring the second and higher power of $A_{2}$, we take equations of motion as

$$
\begin{aligned}
& \ddot{\xi}-2 n \dot{\eta}=\frac{\partial W}{\partial \xi}-\frac{d}{d t}\left(\frac{\partial W}{\partial \dot{\xi}}\right), \\
& \ddot{\eta}+2 n \dot{\xi}=\frac{\partial W}{\partial \eta}-\frac{d}{d t}\left(\frac{\partial W}{\partial \dot{\eta}}\right),
\end{aligned}
$$

where

$$
\begin{aligned}
W & =\frac{1}{2}\left(1+\frac{3 A_{2}}{2}\right)\left(\xi^{2}+\eta^{2}\right)+\frac{q_{1}(1-\mu)}{\rho_{1}}+\frac{\mu}{\rho_{2}}(1 \\
& \left.+\frac{A_{2}}{2 \rho_{2}^{2}}\right)+\frac{1}{c^{2}}\left[-\frac{3}{2}\left\{1-\frac{1}{3} \mu(1-\mu)\right\}\left(1+\frac{3 A_{2}}{4}\right)\left(\xi^{2}\right.\right. \\
& \left.+\eta^{2}\right)+\frac{1}{8}\left\{\dot{\xi}^{2}+\dot{\eta}^{2}+\left(2+\frac{3 A_{2}}{2}\right)(\xi \dot{\eta}-\eta \dot{\xi})+(1\right. \\
& \left.\left.+\frac{3 A_{2}}{2}\right)\left(\xi^{2}+\eta^{2}\right)\right\}^{2}+\frac{3}{2}\left(\frac{q_{1}(1-\mu)}{\rho_{1}}+\frac{\mu}{\rho_{2}}(1\right. \\
& \left.\left.+\frac{A_{2}}{2 \rho_{2}^{2}}\right)\right)\left\{\dot{\xi}^{2}+\dot{\eta}^{2}+\left(2+\frac{3 A_{2}}{2}\right)(\xi \dot{\eta}-\eta \dot{\xi})+(1\right. \\
& \left.\left.+\frac{3 A_{2}}{2}\right)\left(\xi^{2}+\eta^{2}\right)\right\}-\frac{1}{2}\left(\frac{q_{1}^{2}(1-\mu)^{2}}{\rho_{1}^{2}}+\frac{\mu^{2}}{\rho_{2}^{2}}(1\right. \\
& \left.\left.+\frac{A_{2}}{2 \rho_{2}^{2}}\right)^{2}\right)+q_{1} \mu(1-\mu)\left\{\left(\left(4+3 A_{2}\right) \dot{\eta}\right.\right. \\
& \left.+\left(\frac{7}{2}+\frac{21}{4} A_{2}\right) \xi\right)\left(\frac{1}{\rho_{1}}-\frac{1}{\rho_{2}}\left(1+\frac{A_{2}}{2 \rho_{2}^{2}}\right)\right) \\
& -\left(\frac{2+3 A_{2}}{4}\right) \eta^{2}\left(\frac{\mu}{\rho_{1}^{3}}+\frac{q_{1}(1-\mu)}{\rho_{2}^{3}}\right)+\left(1+\frac{3 A_{1}}{2}\right) \\
& +\left(\frac{-1}{\rho_{1} \rho_{2}}+\frac{\mu}{2 \rho_{1}}-\frac{q_{1}(1-\mu)}{\rho_{1}}+\frac{q_{1}(1-\mu)}{2 \rho_{2}}\right. \\
& \left.\left.\left.\left(1+\frac{A_{2}}{2 \rho_{2}^{2}}\right)\right)\right\}\right],
\end{aligned}
$$

with the perturbed mean motion

$$
n=1+\frac{3}{4} A_{2}-\frac{3}{2 c^{2}}\left(1-\frac{1}{3} \mu(1-\mu)\right),
$$

where $A_{2}=\left(\left(A E^{2}-A P^{2}\right) / 5 R^{2}\right)$ [36], $A E$ and $A P$ are, respectively, the equatorial and polar radii and the radiation factor $q_{1}$ is given by $F_{P 1}=F_{g 1}\left(1-q_{1}\right)$ such that $0<\left(1-q_{1}\right) \ll$ 1 Radzievskii [15] where $F_{g 1}$ and $F_{P 1}$ are, respectively, the gravitation and radiation pressure force.

\section{Location of Triangular Points}

The libration points can be obtained from (5) after putting $\dot{\xi}=\dot{\eta}=\ddot{\xi}=\ddot{\eta}=0$.

These points are the solutions of the equations:

$$
\frac{\partial W}{\partial \xi}=0=\frac{\partial W}{\partial \eta} \quad \text { with } \dot{\xi}=\dot{\eta}=0 .
$$


For simplicity, putting $q_{1}=1-\left(1-q_{1}\right)$ and neglecting second and higher power of $\left(1-q_{1}\right)$ as $0<\left(1-q_{1}\right)=\delta \ll 1$ and also the product of $A_{2}$ and $\delta$, we obtain

$$
\begin{aligned}
\xi- & \frac{(1-\mu)(\xi+\mu)}{\rho_{1}^{3}}-\frac{\mu(\xi-1+\mu)}{\rho_{2}^{3}}+\frac{3}{2} A_{2}\{\xi \\
& \left.-\frac{\mu(\xi-1+\mu)}{\rho_{2}^{5}}\right\}+\frac{\delta(1-\mu)(\xi+\mu)}{\rho_{1}^{3}} \\
& +\frac{1}{c^{2}}\left[-3 \xi\left(1-\frac{\mu(1-\mu)}{3}\right)+\frac{1}{2} \xi\left(\xi^{2}+\eta^{2}\right)-\frac{3}{2}\left(\xi^{2}\right.\right. \\
& \left.+\eta^{2}\right)\left\{\frac{(1-\mu)(\xi+\mu)}{\rho_{1}^{3}}+\frac{\mu(\xi-1+\mu)}{\rho_{2}^{3}}\right\} \\
& +3\left(\frac{1-\mu}{\rho_{1}}+\frac{\mu}{\rho_{2}}\right) \xi+\frac{(1-\mu)^{2}}{\rho_{1}^{4}}+\frac{\mu^{2}(\xi-1+\mu)}{\rho_{2}^{4}} \\
& +\mu(1-\mu)\left\{\frac{7}{2}\left(\frac{1}{\rho_{1}}-\frac{1}{\rho_{2}}\right)+\frac{7}{2} \xi\left(-\frac{(\xi+\mu)}{\rho_{1}^{3}}\right.\right. \\
& \left.+\frac{(\xi-1+\mu)(-2+4 \mu)}{2 \rho_{2}^{3}}\right\}+A_{2}\left\{-\frac{9}{4}(1\right. \\
& \left.+\frac{(\xi-1+\mu)}{\rho_{2}^{3}}\right)+\frac{3}{2} \eta^{2}\left(\frac{\mu(\xi+\mu)}{\rho_{1}^{5}}\right. \\
& \left.+\frac{(\xi-1+\mu)}{\rho_{2}}\right)+\frac{7}{2} \xi\left(-\frac{(\xi+\mu)}{\rho_{1}^{3} \rho_{2}^{3}}+\frac{(4-5 \mu)(\xi+\mu)}{2 \rho_{1}^{3}}+\frac{(\xi-1+\mu)}{\rho_{2}^{3}}\right) \\
& \left.+\frac{(1-\mu)(\xi-1+\mu)}{\rho_{2}^{5}}\right)+\frac{(\xi-1+\mu)}{\rho_{1} \rho_{2}^{3}}+\frac{(\xi+\mu)}{\rho_{1}^{3} \rho_{2}} \\
& +\left\{\frac{3}{2}\left(\xi^{2}+\eta^{2}\right) \frac{(1-\mu)(\xi+\mu)}{\rho_{1}^{3}}-\frac{3(1-\mu) \xi}{\rho_{1}}\right. \\
& \left.+\frac{(3 \mu-2)(\xi+\mu)}{2 \rho_{1}^{3}}-\frac{(1-3 \mu)(\xi-1+\mu)}{2 \rho_{2}^{3}}\right\}
\end{aligned}
$$

$$
\begin{aligned}
& \left.+\left(\frac{3 \mu}{2 \rho_{2}^{3}}+\frac{9 \mu}{2 \rho_{2}}+\frac{9(1-\mu)}{2 \rho_{1}}\right) \xi+\frac{2 \mu^{2}(\xi-1+\mu)}{\rho_{2}^{6}}\right\} \\
& +\mu(1-\mu) A_{2}\left\{-\frac{7}{4 \rho_{2}^{3}}+\frac{21}{4}\left(\frac{1}{\rho_{1}}-\frac{1}{\rho_{2}}\right)+\frac{7}{2}\right. \\
& . \xi\left(\frac{3(\xi-1+\mu)}{2 \rho_{2}^{5}}\right)+\frac{21}{4} \xi\left(-\frac{(\xi+\mu)}{\rho_{1}^{3}}\right. \\
& \left.+\frac{(\xi-1+\mu)}{\rho_{2}^{3}}\right)+\frac{9}{4} \eta^{2}\left(\frac{\mu(\xi+\mu)}{\rho_{1}^{5}}\right. \\
& \left.+\frac{(1-\mu)(\xi-1+\mu)}{\rho_{2}^{5}}\right)+\frac{3 \mu(\xi-1+\mu)}{2 \rho_{2}^{5}} \\
& +\frac{3(\xi+\mu)}{2 \rho_{1}^{3} \rho_{2}}+\frac{3(\xi-1+\mu)}{2 \rho_{1} \rho_{2}^{3}}-\frac{3(3 \mu-2)(\xi+\mu)}{4 \rho_{1}^{3}} \\
& \left.\left.-\frac{3(1-\mu)(\xi-1+\mu)}{4 \rho_{2}^{3}}+\frac{3 \mu(\xi-1+\mu)}{2 \rho_{2}^{3}}\right\}\right]=0
\end{aligned}
$$$$
\eta F=0
$$

with

$$
\begin{aligned}
& F=\left(1-\frac{(1-\mu)}{\rho_{1}^{3}}-\frac{\mu}{\rho_{2}^{3}}+\frac{3}{2} A_{2}-\frac{3 \mu}{2 \rho_{2}^{5}} A_{2}\right. \\
& \left.+\frac{\delta(1-\mu)}{\rho_{1}^{3}}\right)+\frac{1}{c^{2}}\left[-3\left(1-\frac{\mu(1-\mu)}{3}\right)+\frac{1}{2}\left(\xi^{2}\right.\right. \\
& \left.+\eta^{2}\right)+3\left(\frac{1-\mu}{\rho_{1}}+\frac{\mu}{\rho_{2}}\right)-\frac{3}{2}\left(\xi^{2}+\eta^{2}\right)\left(\frac{1-\mu}{\rho_{1}^{3}}\right. \\
& \left.+\frac{\mu}{\rho_{2}^{3}}\right)+\left(\frac{(1-\mu)^{2}}{\rho_{1}^{4}}+\frac{\mu^{2}}{\rho_{2}^{4}}\right)+\mu(1-\mu)\left\{\frac{7}{2}\right. \\
& \cdot \xi\left(-\frac{1}{\rho_{1}^{3}}+\frac{1}{\rho_{2}^{3}}\right)-\left(\frac{\mu}{\rho_{1}^{3}}+\frac{1-\mu}{\rho_{2}^{3}}\right)+\frac{3}{2} \eta^{2}\left(\frac{\mu}{\rho_{1}^{5}}\right. \\
& \left.\left.+\frac{1-\mu}{\rho_{2}^{5}}\right)+\frac{1}{\rho_{1}^{3} \rho_{2}}+\frac{1}{\rho_{1} \rho_{2}^{3}}-\frac{(3 \mu-2)}{2 \rho_{1}^{3}}-\frac{(1-3 \mu)}{2 \rho_{2}^{3}}\right\} \\
& +\left\{-\frac{3(1-\mu)}{\rho_{1}}+\frac{3}{2}\left(\xi^{2}+\eta^{2}\right) \frac{(1-\mu)}{\rho_{1}^{3}}\right. \\
& \left.-\frac{2(1-\mu)^{2}}{\rho_{1}^{4}}\right\} \delta-\mu(1-\mu) \delta\left\{\frac{7}{2} \xi\left(-\frac{1}{\rho_{1}^{3}}+\frac{1}{\rho_{2}^{3}}\right)\right. \\
& -\frac{\mu}{\rho_{1}^{3}}-\frac{2(1-\mu)}{\rho_{2}^{3}}+3 \eta^{2}\left(\frac{\mu}{2 \rho_{1}^{5}}+\frac{1-\mu}{\rho_{2}^{5}}\right)\left(\frac{1}{\rho_{1}^{3} \rho_{2}}\right. \\
& \left.\left.+\frac{1}{\rho_{1} \rho_{2}^{3}}+\frac{(4-5 \mu)}{2 \rho_{1}^{3}}+\frac{(4 \mu-2)}{2 \rho_{2}^{3}}\right)\right\}+A_{2}\left\{-\frac{9}{4}(1\right.
\end{aligned}
$$




$$
\begin{aligned}
& \left.-\frac{\mu(1-\mu)}{3}\right)+\frac{3}{2}\left(\xi^{2}+\eta^{2}\right)+\left(-\frac{9(1-\mu)}{4 \rho_{1}^{3}}-\frac{9 \mu}{4 \rho_{2}^{3}}\right. \\
& \left.-\frac{9 \mu}{4 \rho_{2}^{5}}\right)\left(\xi^{2}+\eta^{2}\right)+\frac{3 \mu}{2 \rho_{2}^{3}}+\frac{9(1-\mu)}{2 \rho_{1}}+\frac{9 \mu}{2 \rho_{2}} \\
& \left.+\frac{2 \mu^{2}}{\rho_{2}^{6}}\right\}+\mu(1-\mu) A_{2}\left\{\frac{21}{4} \xi\left(\frac{1}{\rho_{2}^{5}}+\frac{1}{\rho_{2}^{3}}-\frac{1}{\rho_{1}^{3}}\right)\right. \\
& -\frac{3}{2}\left(\frac{\mu}{\rho_{1}^{3}}+\frac{1-\mu}{\rho_{2}^{3}}\right)+\frac{9}{4} \eta^{2}\left(\frac{\mu}{\rho_{1}^{5}}+\frac{1-\mu}{\rho_{2}^{5}}\right) \\
& +\frac{3}{2}\left(\frac{\mu}{\rho_{2}^{5}}+\frac{\mu}{\rho_{2}^{3}}+\frac{1}{\rho_{1}^{3} \rho_{2}}+\frac{1}{\rho_{1} \rho_{2}^{3}}-\frac{(3 \mu-2)}{2 \rho_{1}^{3}}\right. \\
& \left.\left.\left.-\frac{(1-\mu)}{2 \rho_{2}^{3}}\right)\right\}\right] .
\end{aligned}
$$

The triangular points are the solutions of (9) with $\eta \neq 0$.

Since $1 / c^{2} \ll 1$ and in the case $1 / c^{2} \rightarrow 0$ and in the absence of oblateness and radiation (i.e., $A_{2}=\delta=0$ ), one can obtain $\rho_{1}=\rho_{2}=1$, and we assume in the relativistic $\mathrm{R} 3 \mathrm{BP}$ with radiation pressure and oblateness that $\rho_{1}=1+x$ and $\rho_{2}=1+y$ where $x, y \ll 1$ may be depending upon the radiation, oblateness, and relativistic terms. Substituting these values in (4), solving them for $\xi, \eta$, and ignoring terms of second and higher powers of $x$ and $y$, we get

$$
\begin{aligned}
& \xi=x-y+\frac{1-2 \mu}{2}, \\
& \eta= \pm\left(\frac{\sqrt{3}}{2}+\frac{x+y}{\sqrt{3}}\right) .
\end{aligned}
$$

Now substituting the values of $\rho_{1}, \rho_{2}, \xi, \eta$ from the above equations (9) with $\eta \neq 0$ and neglecting second and higher terms in, $y, 1 / c^{2}, A_{2}, \delta$, we have

$$
\begin{aligned}
& \left\{\frac{3}{2}(1-\mu)+\frac{3}{2} A_{2}(1-\mu)+\frac{\delta}{2}(\mu-1)\right\} x-\left\{\frac{3 \mu}{2}\right. \\
& \left.+\frac{3}{2}\left(1+\frac{3}{2} \mu\right) A_{2}+\delta(1-\mu)\right\} y+\frac{3}{4} A_{2}(1-\mu) \\
& +\frac{\delta}{2}(1-\mu)+\frac{1}{c^{2}}\left\{\left(-\frac{9 \mu}{16}+\frac{27 \mu^{2}}{16}-\frac{9 \mu^{3}}{8}\right)\right. \\
& +\left(-\frac{7}{4}+\frac{51 \mu}{8}-\frac{109 \mu^{2}}{16}+\frac{35 \mu^{3}}{16}\right) \delta \\
& \left.+\left(\frac{3}{4}-\frac{137 \mu}{32}+\frac{195 \mu^{2}}{32}-\frac{63 \mu^{3}}{16}\right) A_{2}\right\}=0,
\end{aligned}
$$

$$
\begin{aligned}
& \{3(1-\mu)+3(\mu-1) \delta\} x+\left\{3 \mu+\frac{15}{2} \mu A_{2}\right\} y+\frac{3}{2} \\
& \cdot A_{2}(1-\mu)+(1-\mu) \delta+\frac{1}{c^{2}}\left\{\frac{21}{8} \mu(1-\mu)\right. \\
& +\left(\frac{3}{2}+\frac{117 \mu}{16}-\frac{121 \mu^{2}}{16}+\frac{3 \mu^{3}}{2}\right) A_{2} \\
& \left.+\left(-\frac{7}{2}+\frac{3 \mu}{4}+\frac{39 \mu^{2}}{8}-\frac{17 \mu^{3}}{8}\right) \delta\right\}=0 .
\end{aligned}
$$

Solving these equations for $x$ and $y$, we get

$$
\begin{aligned}
x= & -\frac{\mu(2+3 \mu)}{8 c^{2}}-\left\{\frac{1}{2}+\frac{39-16 \mu+51 \mu^{2}}{48 c^{2}}\right\} A_{2} \\
& +\left\{-\frac{1}{3}+\frac{1}{24 c^{2}}(23-22 \mu)\right\} \delta, \\
y= & -\frac{(1-\mu)(5-3 \mu)}{8 c^{2}} \\
& -\left\{\frac{-15+97 \mu-68 \mu^{2}+30 \mu^{3}}{48 \mu c^{2}}\right\} A_{2} \\
& +\left\{\frac{1}{24 c^{2}}\left(33-64 \mu+\frac{5}{\mu}+26 \mu^{2}\right)\right\} \delta .
\end{aligned}
$$

Thus, the coordinates of the triangular points $(\xi, \pm \eta)$ denoted by $L_{4}$ and $L_{5}$, respectively, are

$$
\begin{aligned}
\xi= & \frac{1-2 \mu}{2}\left(1+\frac{5}{4 c^{2}}\right)+\left\{-\frac{1}{2}\right. \\
& \left.-\frac{15-58 \mu+52 \mu^{2}+21 \mu^{3}}{48 \mu c^{2}}\right\} A_{2}-\left\{\frac{1}{3}\right. \\
& \left.+\frac{1}{8 c^{2}}\left(\frac{10}{3}-14 \mu+\frac{5}{3 \mu}+\frac{26 \mu^{2}}{3}\right)\right\} \delta, \\
\eta & +\left\{\frac{\sqrt{3}}{2}\left(1+\frac{1}{12 c^{2}}\left(-5+6 \mu-6 \mu^{2}\right)\right)\right. \\
& +\left(-\frac{\sqrt{3}}{6}-\frac{\sqrt{3}\left(-15+136 \mu-84 \mu^{2}+81 \mu^{3}\right)}{144 \mu c^{2}}\right) A_{2} \\
& \left.+\frac{\sqrt{3}}{9}\left(-1+\frac{1}{4 c^{2}}\left(28-43 \mu+\frac{5}{2 \mu}+13 \mu^{2}\right)\right) \delta\right\} .
\end{aligned}
$$




\section{Stability of $L_{4}$}

Let $(a, b)$ be the coordinates of the triangular points $L_{4}$.

We set $\xi=a+\alpha, \eta=b+\beta,(\alpha, \beta \ll 1)$, in (5) of motion.

First, we compute the terms of their R.H.S, neglecting second and higher order terms of $A_{2}, \delta$, and their products. We have

$$
\left(\frac{\partial W}{\partial \xi}\right)_{\xi=a+\alpha, \eta=b+\beta}=A \alpha+B \beta+C \dot{\alpha}+D \dot{\beta}
$$

where

$$
\begin{aligned}
A= & \frac{3}{4}\left\{1+\frac{1}{2 c^{2}}\left(2-19 \mu+19 \mu^{2}\right)\right\}+\left\{\frac{3+24 \mu}{8}\right. \\
& \left.+\frac{-30+367 \mu-915 \mu^{2}+717 \mu^{3}+87 \mu^{4}}{32 \mu c^{2}}\right\} A_{2} \\
& +\left\{\frac { 1 } { 2 4 \mu c ^ { 2 } } \left(-30-61 \mu+444 \mu^{2}-615 \mu^{3}\right.\right. \\
& \left.\left.+234 \mu^{4}\right)\right\} \delta, \\
B= & \frac{3 \sqrt{3}}{4}(1-2 \mu)\left(1-\frac{2}{3 c^{2}}\right)+\left\{-\frac{\sqrt{3}}{8}(26 \mu-7)\right. \\
& \left.+\frac{\sqrt{3}\left(30-458 \mu+1498 \mu^{2}-1533 \mu^{3}+417 \mu^{4}\right)}{96 \mu c^{2}}\right\} \\
& +\left\{\frac{2}{3 c^{2}}\left(-5+9 \mu-6 \mu^{2}\right)\right\} \delta . \\
& +\frac{6-5 \mu+5 \mu^{2}}{2 c^{2}}(1-2 \mu)+\left\{-\frac{\sqrt{3}(46 \mu-11)}{24 c^{2}}\right\} A_{2} \\
& \left.\left.-9 \mu^{3}+40 \mu^{4}\right)\right\} \delta, \\
& +\frac{\sqrt{3}}{6}\left\{-(1+\mu)+\frac{1}{8 \mu c^{2}}\left(-10-26 \mu+101 \mu^{2}\right.\right.
\end{aligned}
$$

Similarly, we obtain

$$
\left(\frac{\partial W}{\partial \eta}\right)_{\xi=a+\alpha, \eta=b+\beta}=A_{1} \alpha+B_{1} \beta+C_{1} \dot{\alpha}+D_{1} \dot{\beta}
$$

where

$$
\begin{aligned}
& A_{1}=\frac{3 \sqrt{3}}{4}(1-2 \mu)\left(1-\frac{2}{3 c^{2}}\right)-\left\{\frac{\sqrt{3}(26 \mu-7)}{8}\right. \\
& \left.+\frac{\sqrt{3}\left(30-458 \mu+1498 \mu^{2}-1533 \mu^{3}+417 \mu^{4}\right)}{96 \mu c^{2}}\right\} \\
& \cdot A_{2}+\left\{-\frac{\sqrt{3}(1+\mu)}{6}+\frac{1}{2 c^{2}}\left(-\frac{13 \sqrt{3}}{12}+\frac{101 \sqrt{3}}{24} \mu\right.\right. \\
& \left.\left.-\frac{3 \sqrt{3}}{8} \mu^{2}-\frac{5 \sqrt{3}}{12 \mu}+\frac{5 \sqrt{3}}{3} \mu^{3}\right)\right\} \delta \\
& B_{1}=\frac{9}{4}\left\{1+\frac{7}{3 c^{2}}\left(-2+3 \mu-3 \mu^{2}\right)\right\}+\left\{\frac{33}{8}\right. \\
& \left.+\frac{30-347 \mu+915 \mu^{2}-777 \mu^{3}+111 \mu^{4}}{32 \mu c^{2}}\right\} A_{2} \\
& +\left\{\frac{1}{2}(1-3 \mu)+\frac{1}{4 c^{2}}\left(-\frac{45}{4}-11 \mu+\frac{5}{2 \mu}+\frac{193}{4} \mu^{2}\right.\right. \\
& \left.\left.-\frac{59}{2} \mu^{3}\right)\right\} \delta \\
& C_{1}=\frac{1}{2 c^{2}}\left(-4+\mu-\mu^{2}\right)-\left\{\frac{\left(16-5 \mu+9 \mu^{2}\right)}{8 c^{2}}\right\} A_{2} \\
& +\left\{\frac{1}{3 c^{2}}(5-7 \mu)\right\} \delta \\
& D_{1}=-\frac{\sqrt{3}(1-2 \mu)}{2 c^{2}}+\left\{\frac{\sqrt{3}(-11+46 \mu)}{24 c^{2}}\right\} A_{2} \\
& +\left\{\frac{\sqrt{3}}{9 c^{2}}\left(4-20 \mu+18 \mu^{2}\right)\right\} \delta, \\
& \frac{d}{d t}\left(\frac{\partial W}{\partial \dot{\xi}}\right)_{\xi=a+\alpha, \eta=b+\beta}=F \dot{\alpha}+B_{2} \dot{\beta}+C_{2} \ddot{\alpha}+D_{2} \ddot{\beta},
\end{aligned}
$$

where

$$
\begin{aligned}
F= & \frac{\sqrt{3}}{2 c^{2}}(1-2 \mu) \\
& -\left\{\frac{\sqrt{3}(46 \mu-11)}{24 c^{2}}\right\} A_{2} \\
& +\left\{\frac{2 \sqrt{3}}{9 c^{2}}(\mu-2)\right\} \delta, \\
B_{2}= & -\frac{4-\mu+\mu^{2}}{2 c^{2}}
\end{aligned}
$$


TABLE 1: Locations of triangular points for the Sun-Mars system: $c=12424.24, \mu=0.000000322700$, and $A_{2}=0.000000001 \times 10^{-8}$.

\begin{tabular}{lcccccc}
\hline$\delta$ & Classical & $\begin{array}{c}\xi \\
\text { Relativistic }\end{array}$ & Equation (14) & Classical & $\begin{array}{c} \pm \eta \\
\text { Relativistic }\end{array}$ & Equation (14) \\
\hline 0.0 & 0.4999996773 & 0.4999996813 & 0.4999996813 & 0.8660254040 & 0.8660254016 & 0.8660254016 \\
0.0001 & 0.4999996773 & 0.4999996813 & 0.4999659297 & 0.8660254040 & 0.8660254016 & 0.8660063980 \\
0.001 & 0.4999996773 & 0.4999996813 & 0.4996621656 & 0.8660254040 & 0.8660254016 & 0.8658353662 \\
0.01 & 0.4999996773 & 0.4999996813 & 0.4966245245 & 0.8660254040 & 0.8660254016 & 0.8641250476 \\
0.02 & 0.4999996773 & 0.4999996813 & 0.4932493676 & 0.8660254040 & 0.8660254016 & 0.8622246936 \\
\hline
\end{tabular}

TABLE 2: Critical Mass for the Sun-Mars system: $c=12424.24, \mu=$ 0.000000322700 , and $A_{2}=0.0000000001 \times 10^{-8}$.

\begin{tabular}{|c|c|c|c|}
\hline$\delta$ & Classical & $\begin{array}{c}\mu_{c} \\
\text { Relativistic }\end{array}$ & Equation (34) \\
\hline 0.0 & 0.0385208965 & 0.0385208946 & 0.0385208946 \\
\hline 0.0001 & 0.0385208965 & 0.0385208946 & 0.0385200028 \\
\hline 0.001 & 0.0385208965 & 0.0385208946 & 0.0385119771 \\
\hline 0.01 & 0.0385208965 & 0.0385208946 & 0.0384317199 \\
\hline 0.02 & 0.0385208965 & 0.0385208946 & 0.0383425452 \\
\hline
\end{tabular}

$$
\begin{aligned}
& -\left\{\frac{16-5 \mu+9 \mu^{2}}{8 c^{2}}\right\} A_{2} \\
& +\left\{\frac{1}{3 c^{2}}(5-7 \mu)\right\} \delta, \\
C_{2}= & \frac{17-2 \mu+2 \mu^{2}}{4 c^{2}} \\
& +\left\{\frac{19-2 \mu+6 \mu^{2}}{8 c^{2}}\right\} A_{2} \\
& +\left\{\frac{1}{3 c^{2}}(-8+7 \mu)\right\} \delta, \\
D_{2}= & -\frac{\sqrt{3}}{4 c^{2}}(1-2 \mu) \\
& +\left\{\frac{\sqrt{3}(-1+14 \mu)}{24 c^{2}}\right\} A_{2} \\
& +\left\{\frac{\sqrt{3}}{9 c^{2}}(2-\mu)\right\} \delta, \\
\frac{d}{d t}\left(\frac{\partial W}{\partial \dot{\eta}}\right)_{\xi=a+\alpha, \eta=b+\beta} & A_{3} \dot{\alpha}+B_{3} \dot{\beta}+C_{3} \ddot{\alpha}+D_{3} \ddot{\beta},
\end{aligned}
$$

where

$$
\begin{aligned}
A_{3}= & \frac{6-5 \mu+5 \mu^{2}}{2 c^{2}}+\left\{\frac{34-57 \mu+45 \mu^{2}}{8 c^{2}}\right\} A_{2} \\
& +\left\{\frac{1}{3 c^{2}}\left(-5+9 \mu-6 \mu^{2}\right)\right\} \delta,
\end{aligned}
$$

$$
\begin{aligned}
B_{3}= & -\frac{\sqrt{3}(1-2 \mu)}{2 c^{2}}+\left\{\frac{\sqrt{3}(-11+46 \mu)}{24 c^{2}}\right\} A_{2} \\
& +\left\{\frac{2 \sqrt{3}}{9 c^{2}}\left(2-10 \mu+9 \mu^{2}\right)\right\} \delta, \\
C_{3}= & -\frac{\sqrt{3}(1-2 \mu)}{4 c^{2}}+\left\{\frac{\sqrt{3}(-1+14 \mu)}{24 c^{2}}\right\} A_{2} \\
& +\left\{\frac{\sqrt{3}}{9 c^{2}}(2-\mu)\right\} \delta, \\
D_{3}= & \frac{3\left(5-2 \mu+2 \mu^{2}\right)}{4 c^{2}}+\left\{\frac{13-6 \mu+18 \mu^{2}}{8 c^{2}}\right\} A_{2} \\
& +\left\{\frac{1}{3 c^{2}}(9 \mu-8)\right\} \delta .
\end{aligned}
$$

Thus, the variational equations of motion corresponding to (5), on making use of (7), can be obtained as

$$
\begin{aligned}
& P_{1} \ddot{\alpha}+P_{2} \ddot{\beta}+P_{3} \dot{\alpha}+P_{4} \dot{\beta}+P_{5} \alpha+P_{6} \beta=0, \\
& q_{1} \ddot{\alpha}+q_{2} \ddot{\beta}+q_{3} \dot{\alpha}+q_{4} \dot{\beta}+q_{5} \alpha+q_{6} \beta=0,
\end{aligned}
$$

where

$$
\begin{aligned}
P_{1} & =1+C_{2}, \\
P_{2} & =D_{2}, \\
P_{3} & =F-C, \\
P_{4} & =\left\{B_{2}-2\left(1+\frac{3}{4} A_{2}-\frac{3}{2 c^{2}}\left(1-\frac{1}{3} \mu(1-\mu)\right)\right)\right. \\
& -D\}, \\
P_{5} & =-A, \\
P_{6} & =-B, \\
q_{1} & =C_{3},
\end{aligned}
$$


TABLE 3: Locations of triangular points. Sun-Jupiter system: $c=22247.35, \mu=0.000953692200$, and $A_{2}=0.0000192887 \times 10^{-8}$.

\begin{tabular}{lccccrc}
\hline$\delta$ & Classical & $\xi$ & & & $\pm \eta$ \\
Relativistic & Equation (14) & Classical & Relativistic & Equation (14) \\
\hline 0.0 & 0.4990463078 & 0.4990463093 & 0.4990463093 & 0.8660254040 & 0.8660254033 & 0.8660254033 \\
0.0001 & 0.4990463078 & 0.4990463093 & 0.4990129759 & 0.8660254040 & 0.8660254033 & 0.8660061583 \\
0.001 & 0.4990463078 & 0.4990463093 & 0.4987129755 & 0.8660254040 & 0.8660254033 & 0.8658329534 \\
0.01 & 0.4990463078 & 0.4990463093 & 0.4957129715 & 0.8660254040 & 0.8660254033 & 0.8641009050 \\
0.02 & 0.4990463078 & 0.4990463093 & 0.4923796338 & 0.8660254040 & 0.8660254033 & 0.8621764067 \\
\hline
\end{tabular}

TABle 4: Critical Mass. Sun-Mars system: $c=22247.35, \mu=$ 0.000000322700 , and $A_{2}=0.0000000001 \times 10^{-8}$.

\begin{tabular}{lccc}
\hline$\delta$ & \multicolumn{3}{c}{$\mu_{c}$} \\
& Classical & Relativistic & Equation (34) \\
\hline 0.0 & 0.0385208965 & 0.0385208959 & 0.0385208959 \\
0.0001 & 0.0385208965 & 0.0385208959 & 0.0385200042 \\
0.001 & 0.0385208965 & 0.0385208959 & 0.0385119784 \\
0.01 & 0.0385208965 & 0.0385208959 & 0.0384317212 \\
0.02 & 0.0385208965 & 0.0385208959 & 0.0383425464 \\
\hline
\end{tabular}

$$
\begin{aligned}
q_{2} & =1+D_{3} \\
q_{3} & =2\left(1+\frac{3}{4} A_{2}-\frac{3}{2 c^{2}}\left(1-\frac{1}{3} \mu\left(1+\mu^{2}\right)\right)\right)-C_{1} \\
& +A_{3} \\
q_{4} & =B_{3}-D_{1} \\
q_{5} & =-A_{1} \\
q_{6} & =-B_{1} .
\end{aligned}
$$

Then, the characteristic equation is

$$
\begin{aligned}
\left(P_{1} q_{2}\right. & \left.-P_{2} q_{1}\right) \lambda^{4} \\
& +\left(P_{1} q_{6}+P_{5} q_{2}+P_{3} q_{4}-P_{6} q_{1}-P_{2} q_{5}-P_{4} q_{3}\right) \lambda^{2} \\
& +P_{5} q_{6}-P_{6} q_{5}=0 .
\end{aligned}
$$

Substituting the values of $P_{i}, q_{i}, i=1,2, \ldots, 6$ in (25), the characteristic equation (25) after normalizing becomes

$$
\lambda^{4}+b \lambda^{2}+d=0
$$

where

$$
\begin{aligned}
b & =\left(1-\frac{9}{c^{2}}\right)+\left\{\frac{3}{2}-3 \mu\right. \\
& \left.+\frac{-101-48 \mu+51 \mu^{2}+18 \mu^{3}}{8 c^{2}}\right\} A_{2}
\end{aligned}
$$

$$
\begin{aligned}
& +\left\{\frac{61-6 \mu-57 \mu^{2}+12 \mu^{3}}{12 c^{2}}\right\} \delta, \\
d & =\left\{\frac{27 \mu(1-\mu)}{4}\right. \\
& \left.+\frac{9 \mu\left(-65+77 \mu-24 \mu^{2}+12 \mu^{3}\right)}{8 c^{2}}\right\} \\
& +\left\{\frac{117}{4} \mu(1-\mu)\right. \\
& \left.+\frac{3\left(-61-5289 \mu+4602 \mu^{2}-18 \mu^{3}+846 \mu^{4}\right)}{64 c^{2}}\right\} \\
& +A_{2}+\left\{\frac{3}{2} \mu(1-\mu)\right. \\
+ & \left.\frac{-61+397 \mu-832 \mu^{2}+240 \mu^{3}+336 \mu^{4}}{32 c^{2}}\right\} \delta .
\end{aligned}
$$

When $1 / c^{2} \rightarrow 0$ and in the absence of the oblateness and radiation (i.e., $A_{1}=\delta=0$ ), (26) reduces to its well-known classical restricted problem form (see, e.g., [10]):

$$
\lambda^{4}+\lambda^{2}+\frac{27 \mu(1-\mu)}{4}=0 .
$$

The discriminant of (26) is

$$
\begin{aligned}
\Delta & =\left(\frac{-54}{c^{2}}-\frac{1269}{8 c^{2}} A_{2}-\frac{42}{c^{2}} \delta\right) \mu^{4}+\left(\frac{108}{c^{2}}+\frac{63}{8 c^{2}} A_{2}\right. \\
& \left.-\frac{28}{c^{2}} \delta\right) \mu^{3}+\left(27+117 A_{2}+6 \delta-\frac{693}{2 c^{2}}-\frac{6801}{8 c^{2}} A_{2}\right. \\
& \left.+\frac{189}{2 c^{2}} \delta\right) \mu^{2}+\left(-27-123 A_{2}-6 \delta+\frac{585}{2 c^{2}}\right. \\
& \left.+\frac{16539}{16 c^{2}} A_{2}-\frac{405}{8 c^{2}} \delta\right) \mu+1+3 A_{2}-\frac{18}{c^{2}}-\frac{653 A_{2}}{16 c^{2}} \\
& +\frac{427}{24 c^{2}} \delta .
\end{aligned}
$$


TABLE 5: Locations of triangular points. Sun-Saturn system: $c=31050.90, \mu=0.000285726000$, and $A_{2}=0.0000018690 \times 10^{-8}$.

\begin{tabular}{lcccccc}
\hline$\delta$ & $\begin{array}{c}\xi \\
\text { Classical }\end{array}$ & Relativistic & Equation (14) & Classical & $\begin{array}{c} \pm \eta \\
\text { Relativistic }\end{array}$ & \begin{tabular}{c} 
Equation (14) \\
\hline 0.0
\end{tabular} \\
0.0001 & 0.4997142740 & 0.4997142745 & 0.4997142745 & 0.8660254040 & 0.8660254037 & 0.8660254037 \\
0.001 & 0.4997142740 & 0.4997142745 & 0.4993809404 & 0.8660254040 & 0.8660254037 & 0.8658329539 \\
0.01 & 0.4997142740 & 0.4997142745 & 0.4963809336 & 0.8660254040 & 0.8660254037 & 0.8641009071 \\
0.02 & 0.4997142740 & 0.4997142745 & 0.4930475927 & 0.8660254040 & 0.8660254037 & 0.8621764106 \\
\hline
\end{tabular}

TABle 6: Critical Mass. Sun-Saturn system: $c=31050.90, \mu=$ 0.00028572600 , and $A_{2}=0.0000018690 \times 10^{-8}$.

\begin{tabular}{lccc}
\hline$\delta$ & \multicolumn{3}{c}{$\begin{array}{c}\mu_{c} \\
\text { Relativistic }\end{array}$} \\
\hline 0.0 & 0.0385208965 & 0.0385208961 & 0.0385208961 \\
0.0001 & 0.0385208965 & 0.0385208961 & 0.0385200044 \\
0.001 & 0.0385208965 & 0.0385208961 & 0.0385119786 \\
0.01 & 0.0385208965 & 0.0385208961 & 0.0384317215 \\
0.02 & 0.0385208965 & 0.0385208961 & 0.0383425467 \\
\hline
\end{tabular}

Its roots are

$$
\lambda^{2}=\frac{-b \pm \sqrt{\Delta}}{2}
$$

where

$$
\begin{aligned}
b= & \left(1-\frac{9}{c^{2}}\right) \\
& +\left\{\frac{3}{2}-3 \mu+\frac{-101-48 \mu+51 \mu^{2}+18 \mu^{3}}{8 c^{2}}\right\} A_{2} \\
& +\left\{\frac{61-6 \mu-57 \mu^{2}+12 \mu^{3}}{12 c^{2}}\right\} \delta .
\end{aligned}
$$

From (29), we have

$$
\begin{aligned}
\frac{d \Delta}{d \mu} & =4\left(\frac{-54}{c^{2}}-\frac{1269}{8 c^{2}} A_{2}-\frac{42}{c^{2}} \delta\right) \mu^{3} \\
& +3\left(\frac{108}{c^{2}}+\frac{63}{8 c^{2}} A_{2}-\frac{28}{c^{2}} \delta\right) \mu^{2} \\
& +2\left(27+117 A_{2}+6 \delta-\frac{693}{2 c^{2}}-\frac{6801}{8 c^{2}} A_{2}+\frac{189}{2 c^{2}} \delta\right) \\
& +\mu \\
& +\left(-27-123 A_{2}-6 \delta+\frac{585}{2 c^{2}}+\frac{1653}{16 c^{2}} A_{2}-\frac{405}{8 c^{2}} \delta\right) \\
& <0 \quad \forall \mu \in\left(0, \frac{1}{2}\right] .
\end{aligned}
$$

From (32), it follows that $\Delta$ is decreasing in $(0,1 / 2]$.
But

$$
\begin{aligned}
(\Delta)_{\mu=0}= & 1+3 A_{2}-\frac{18}{c^{2}}-\frac{653 A_{2}}{16 c^{2}}+\frac{427}{24 c^{2}} \delta>0 \\
(\Delta)_{\mu=1 / 2}= & -\frac{23}{4}-\frac{117 A_{2}}{4}-\frac{3}{2} \delta+\frac{207}{4 c^{2}}+\frac{32585 A_{2}}{128 c^{2}} \\
& +\frac{479}{48 c^{2}} \delta<0 .
\end{aligned}
$$

Since $(\Delta)_{\mu=0}$ and $(\Delta)_{\mu=1 / 2}$ are of opposite signs, and $\Delta$ is monotone continuous, there is one value of $\mu$, for example, $\mu_{c}$ in the interval $(0,1 / 2]$ for which $\Delta$ vanishes. Solving the equation $\Delta=0$, using (29), we obtain critical value of the mass parameter as

$$
\begin{aligned}
\mu_{c}= & \frac{1}{2}-\frac{1}{18} \sqrt{69}+\frac{1}{9}\left(1-\frac{13}{\sqrt{69}}\right) A_{2}-\frac{2}{27 \sqrt{69}} \delta \\
& -\frac{17 \sqrt{69}}{486 c^{2}}+\left(\frac{197133+15493 \sqrt{69}}{536544 c^{2}}\right) A_{2} \\
& +\left(\frac{327543+20267 \sqrt{69}}{804816 c^{2}}\right) \delta, \\
\mu_{c}= & \mu_{0}-\frac{17 \sqrt{69}}{486 c^{2}}+\frac{1}{9}\left(1-\frac{13}{\sqrt{69}}\right) A_{2}-\frac{2}{27 \sqrt{69}} \delta \\
& +\left(\frac{197133+15493 \sqrt{69}}{536544 c^{2}}\right) A_{2} \\
& +\left(\frac{327543+20267 \sqrt{69}}{804816 c^{2}}\right) \delta,
\end{aligned}
$$

where $\mu_{0}=0.03852 \ldots$ is Routh's value.

We consider the following three regions of the values of $\mu$ separately:

(i) When $0 \leq \mu<\mu_{c}$, the values of $\lambda^{2}$ given by (30) are negative and therefore all the four characteristic roots are distinct pure imaginary numbers. Hence, the triangular points are stable.

(ii) When $\mu_{c}<\mu \leq 1 / 2, \Delta<0$, the real parts of the characteristic roots are positive. Therefore, the triangular points are unstable. 
TABLE 7: Locations of triangular points. Sun-Uranus system: $c=44056.13, \mu=0.000043548000$, and $A_{2}=0.0000000070 \times 10^{-8}$.

\begin{tabular}{|c|c|c|c|c|c|c|}
\hline \multirow{2}{*}{$\delta$} & \multicolumn{3}{|c|}{$\xi$} & \multicolumn{3}{|c|}{$\pm \eta$} \\
\hline & Classical & Relativistic & Equation (14) & Classical & Relativistic & Equation (14) \\
\hline 0.0 & 0.4999564520 & 0.4999564525 & 0.4999564525 & 0.8660254040 & 0.8660254038 & 0.8660254038 \\
\hline 0.0001 & 0.4999564520 & 0.4999564525 & 0.4999231189 & 0.8660254040 & 0.8660254038 & 0.8660061590 \\
\hline 0.001 & 0.4999564520 & 0.4999564525 & 0.4996231167 & 0.8660254040 & 0.8660254038 & 0.8658329551 \\
\hline 0.01 & 0.4999564520 & 0.4999564525 & 0.4966230945 & 0.8660254040 & 0.8660254038 & 0.8641009172 \\
\hline 0.02 & 0.4999564520 & 0.4999564525 & 0.4932897365 & 0.8660254040 & 0.8660254038 & 0.8621764305 \\
\hline
\end{tabular}

TABle 8: Critical Mass. Sun-Uranus system: $c=44056.13, \mu=$ 0.000043548000 , and $A_{2}=0.0000000070 \times 10^{-8}$.

\begin{tabular}{lccc}
\hline$\delta$ & \multicolumn{3}{c}{$\mu_{c}$} \\
& Classical & Relativistic & Equation (34) \\
\hline 0.0 & 0.0385208965 & 0.0385208963 & 0.0385208963 \\
0.0001 & 0.0385208965 & 0.0385208963 & 0.0385200046 \\
0.001 & 0.0385208965 & 0.0385208963 & 0.0385119788 \\
0.01 & 0.0385208966 & 0.0385208963 & 0.0384317216 \\
0.02 & 0.0385208965 & 0.0385208963 & 0.0383425469 \\
\hline
\end{tabular}

(iii) When $\mu=\mu_{c}, \Delta=0$, the values of $\lambda^{2}$ given by (30) are the same. This induces instability of the triangular points.

Hence, the stability region is

$$
\begin{aligned}
& 0<\mu \\
& <\mu_{0}-\frac{17 \sqrt{69}}{486 c^{2}}+\frac{1}{9}\left(1-\frac{13}{\sqrt{69}}\right) A_{2}-\frac{2}{27 \sqrt{69}} \delta \\
& \quad+\left(\frac{197133+15493 \sqrt{69}}{536544 c^{2}}\right) A_{2} \\
& \quad+\left(\frac{327543+20267 \sqrt{69}}{804816 c^{2}}\right) \delta .
\end{aligned}
$$

\section{Discussion}

In this section we discuss the triangular libration points in the framework of relativistic restricted three-body problem when the more massive primary body is a source of radiation and the less massive (secondary) body is oblate.

In analogy to classical problem, the positions and critical mass parameter of analogous triangular libration points (14) and (34), respectively, are obtained. It is important to note that these triangular libration points (14) cease to be classical ones. That is, they no longer form equilateral triangles with the primaries since $\rho_{1} \neq \rho_{2} \neq 1$. Rather they form scalene triangles but turn into classical ones in the absence of relativistic, radiation, and oblateness factors. Degenerating the present relativistic R3BP to corresponding models, the results of this study are in accordance with those of Douskos and Perdios [32] but in disagreement with those of Bhatnagar and Hallan [31]. In the absence of relativistic factor, it is observed from (25) and (34) that the results of this study correspond to AbdulRaheem and Singh [20] when the bigger primary is luminous only and the smaller one is oblate only in the absence of small perturbations in the Coriolis and centrifugal forces.

In the absence of relativistic terms, our results are also in agreement with those of Abouelmagd and Sharaf [21] when the linear terms, in coefficient $B_{1}$, are retained only and $B_{2}=$ 0 , where $B_{i}=\bar{J}_{2 i} R_{2}^{2 i}(i=1,2), R_{2}$ is the mean radius, and $\bar{J}_{2 i}(i=1,2)$ are dimensionless coefficients that characterize the size of nonspherical components of the potential of the smaller primary. It is also noticed that, in the absence of oblateness terms, the present results are in agreement with those of Singh and Bello [35]. However, for the locations of triangular points, there is disagreement with those of Katour et al. [34] when the bigger primary is radiating only and the smaller one is oblate only. It is seen from (34) that the effects of relativistic terms, radiation, and oblateness on the linear stability of triangular points are observable. They reduce the size of the stability region independently whereas the joint effect of oblateness and relativistic or radiation and relativistic expands it. This expansion can be explained by the presence of positive coefficients of coupling terms $A_{2} / c^{2}$ and $\delta / c^{2}$.

\section{Numerical Applications}

We now use the above mentioned analysis to locate triangular equilibrium points and compute the values of the critical mass parameters for some of Sun-Planet pairs of our solar system. The necessary data have been borrowed from Ragos et al. [37] and Subbarao and Sharma [13]. These values are shown in Tables 1-10.

\section{Conclusion}

Linear stability of triangular points has been studied when the more massive primary is radiating and the smaller one is an oblate spheroid well. It is discovered that their locations and critical mass are affected by radiation and oblateness factors. It is also observed that both factors have destabilizing behavior; that is, an increase in their values reduces the size of stability region independently. However the joint effect of radiation, oblateness, and relativistic terms expands the size of the stability region. It is also noticed that the expressions for $A, D, A_{2}$, and $C_{2}$ in Bhatnagar and Hallan [31] differ from the present study when the radiation pressure and oblateness are absent. Consequently, the expression of $P_{1}, P_{3}, P_{4}$, and $P_{5}$ and the characteristic equation are also different. This led them 
TABLE 9: Locations of triangular points. Sun-Neptune system: $c=55148.85, \mu=0.000051668900$, and $A_{2}=0.0000000010 \times 10^{-8}$.

\begin{tabular}{|c|c|c|c|c|c|c|}
\hline \multirow{2}{*}{$\delta$} & \multicolumn{3}{|c|}{$\xi$} & \multicolumn{3}{|c|}{$\pm \eta$} \\
\hline & Classical & Relativistic & Equation (14) & Classical & Relativistic & Equation (14) \\
\hline 0.0 & 0.4999483311 & 0.4999483311 & 0.4999483311 & 0.8660254040 & 0.8660254040 & 0.8660254040 \\
\hline 0.0001 & 0.4999483311 & 0.4999483311 & 0.4999149976 & 0.8660254040 & 0.8660254040 & 0.8660061590 \\
\hline 0.001 & 0.4999483311 & 0.4999483311 & 0.4996149964 & 0.8660254040 & 0.8660254040 & 0.8658329546 \\
\hline 0.01 & 0.4999483311 & 0.4999483311 & 0.4966149845 & 0.8660254040 & 0.8660254040 & 0.8641009107 \\
\hline 0.02 & 0.4999483311 & 0.4999483311 & 0.4932816379 & 0.8660254040 & 0.8660254040 & 0.8641009107 \\
\hline
\end{tabular}

TABLE 10: Critical Mass. Sun-Neptune system: $c=55148.85, \mu=$ 0.000051668900 , and $A_{2}=0.0000000010 \times 10^{-8}$.

\begin{tabular}{lccc}
\hline$\delta$ & Classical & $\begin{array}{c}\mu_{c} \\
\text { Relativistic }\end{array}$ & Equation (34) \\
\hline 0.0 & 0.0385208965 & 0.0385208964 & 0.0385208964 \\
0.0001 & 0.0385208965 & 0.0385208964 & 0.0385200047 \\
0.001 & 0.0385208965 & 0.0385208964 & 0.0385119789 \\
0.01 & 0.0385208966 & 0.0385208964 & 0.0384317217 \\
0.02 & 0.0385208965 & 0.0385208964 & 0.0383425469 \\
\hline
\end{tabular}

[31] to infer that triangular points are unstable, contrary to Douskos and Perdios [32] and our results.

Our results are also in disagreement with those of Katour et al. [34]. One major distinction is that the expression of the mean motion $n$ which they used in their study differs from our own. It seems that in their expression for the mean motion. In addition to that they have not included the coupling term $A_{2} / c^{2}$ in their study, while we do. Also, they have not analyzed stability, while we do.

It is observed from Tables 1-10 that the numerical exploration conducted on some of the Sun-Planet pairs reveals that the effect of radiation is prominent in the size of stability region and the positions, while their oblateness effects are comparatively negligible.

\section{Conflict of Interests}

The authors declare that there is no conflict of interests regarding the publication of this paper.

\section{References}

[1] S. I. Newton, The Mathematical Principles of Natural Philosophy, Translated into English by A. Motte, 1729.

[2] H. Poincare, Les methodes nouvelles de la mecanique celeste, Gauthier-Villars, Paris, France, 1892.

[3] A. Chenciner, "Three body problem," Scholarpedia, vol. 2, no. 10, article 2111, 2007.

[4] M. Valtonen and H. Karttunen, The Three-Body Problem, Cambridge University Press, Cambridge, UK, 2006.

[5] G. Renzetti, "Exact geodetic precession of the orbit of a twobody gyroscope in geodesic motion about a third mass," Earth, Moon and Planets, vol. 109, no. 1-4, pp. 55-59, 2012.

[6] K. Nordtvedt Jr., "Testing relativity with laser ranging to the moon," Physical Review, vol. 170, no. 5, pp. 1186-1187, 1968.
[7] L. Iorio, "Orbital motions as gradiometers for post-Newtonian tidal effects," Frontiers in Astronomy and Space Sciences, vol. 1, article 3, 2014.

[8] L. Euler, "De motu rectilineo trium corporum se mutuo attrahention," Novi Commentarii Academiae Scientiarum Imperialis Petropolitanae, vol. 11, p. 144, 1765.

[9] J. L. Lagrange, "Essai sur le probléme des trois corps," Oeuvres, vol. 6, pp. 272-292, 1772.

[10] V. Szebehely, Theory of Orbits. The Restricted Problem of ThreeBodies, Academic Press, New York, NY, USA, 1967.

[11] N. Seto and T. Muto, "Relativistic astrophysics with resonant multiple inspirals," Physical Review D, vol. 81, no. 10, Article ID 103004, 6 pages, 2010.

[12] J. D. Schnittman, "The Lagrange equilibrium points $L_{4}$ and $L_{5}$ in black hole binary system," The Astrophysical Journal, vol. 724, no. 1, p. 39, 2010.

[13] P. V. Subbarao and R. K. Sharma, "A note on the stability of the triangular points of equilibrium in the restricted three-body problem," Astronomy and Astrophysics, vol. 43, no. 3, pp. 381383, 1975.

[14] V. V. Markellos, K. E. Papadakis, and E. A. Perdios, "Non-linear stability zones around triangular equilibria in the plane circular restricted three-body problem with oblateness," Astrophysics and Space Science, vol. 245, no. 1, pp. 157-164, 1996.

[15] V. V. Radzievskii, "The restricted problem of three-bodies taking account of light pressure," Astronomical Journal, vol. 27, pp. 250-256, 1950.

[16] V. V. Radzievskii, "The spatial case of the restricted problem of three radiating and gravitating bodies," vol. 30, pp. 265-273, 1953.

[17] R. K. Sharma, "On linear stability of triangular libration points of the photogravitational restricted three-body problem when the more massive primary is an oblate spheroid," in Sun and Planetary System, W. Frickle and G. Teleki, Eds., vol. 435, pp. 345-436, Reidel Publishing Company, 1982.

[18] J. F. L. Simmons, A. J. C. McDonald, and J. C. Brown, "The restricted 3-body with radiation pressure," Celestial Mechanics, vol. 35, no. 2, pp. 145-187, 1985.

[19] R. K. Sharma, "The linear stability of libration points of the photogravitational restricted three-body problem when the smaller primary is an oblate spheroid," Astrophysics and Space Science, vol. 135, no. 2, pp. 271-281, 1987.

[20] A. AbdulRaheem and J. Singh, "Combined effects of perturbations, radiation, and oblateness on the stability of equilibrium points in the restricted three-body problem," The Astronomical Journal, vol. 131, no. 3, pp. 1880-1885, 2006.

[21] E. I. Abouelmagd and M. A. Sharaf, "The motion around the libration points in the restricted three-body problem with the effect of radiation and oblateness," Astrophysics and Space Science, vol. 344, no. 2, pp. 321-332, 2013. 
[22] A. S. Einstein and J. Grommer, Allgemeine Relativitäts tstheorie und Bewegungsgesetz, vol. 1-2 of Sitzungsberichte der Preussischen Akademie der Wissenschaften, Walter de Gruyter, Berlin, Germany, 1927.

[23] W. de Sitter, "On Einstein's theory of garviation and its astronomical consequences," Monthly Notices of the Royal Astronomical Society, vol. 76, pp. 699-728, 1916.

[24] F. Kottler, "Gravitation und relativitts theorie," Encyklopädie der Mathematischen Wissenschaften, vol. 6, no. 2, article 22a, 1922.

[25] J. Chazy, La Théorie de la Relativité et la Mécanique Céleste, vol. 1, Gauthier-Villars, Paris, France, 1928.

[26] J. Chazy, La theorie dela relativite et la mecanique celeste, vol. 1, Gauthier-Villars, Paris, France, 1930.

[27] A. S. Eddington and G. L. Clark, "The problem of $n$ bodies in general relativity theory," Proceedings of the Royal Society of London A, vol. 166, p. 465, 1938.

[28] E. Krefetz, "Restricted three-body problem in the post-Newtonian approximation," The Astronomical Journal, vol. 72, pp. 471473, 1967.

[29] V. A. Brumberg, Relativistic Celestial Mechanics, Nauka, Moscow, Russia, 1972.

[30] V. A. Brumberg, Essential Relativistic Celestial Mechanics, Hilger, New York, NY, USA, 1991.

[31] K. B. Bhatnagar and P. P. Hallan, "Existence and stability of $L_{4,5}$ in the relativistic restricted three-body problem," Celestial Mechanics and Dynamical Astronomy, vol. 69, no. 3, pp. 271-281, 1998.

[32] C. N. Douskos and E. A. Perdios, "On the stability of equilibrium points in the relativistic restricted three-body problem," Celestial Mechanics, vol. 82, no. 4, pp. 317-321, 2002.

[33] F. A. Abd El-Salam and S. E. Abd El-Bar, "On the triangular equilibrium points in the photogravitational relativistic restricted three-body problem," Astrophysics and Space Science, vol. 349, no. 1, pp. 125-135, 2014.

[34] D. A. Katour, F. A. Abd El-Salam, and M. O. Shaker, "Relativistic restricted three body problem with oblatness and photogravitational corrections to triangular equilibrium points," Astrophysics and Space Science, vol. 351, no. 1, pp. 143-149, 2014.

[35] J. Singh and N. Bello, "Effect of radiation pressure on the stability of $L_{4,5}$ in the relativistic R3BP," Astrophysics and Space Science, vol. 351, no. 2, pp. 483-490, 2014.

[36] S. W. McCuskey, Introduction to Celestial Mechanics, AddisonWesley, New York, NY, USA, 1963.

[37] O. Ragos, E. A. Perdios, V. S. Kalantonis, and M. N. Vrahatis, "On the equilibrium points of the relativistic restricted threebody problem," Nonlinear Analysis: Theory, Methods \& Applications, vol. 47, no. 5, pp. 3413-3418, 2001. 

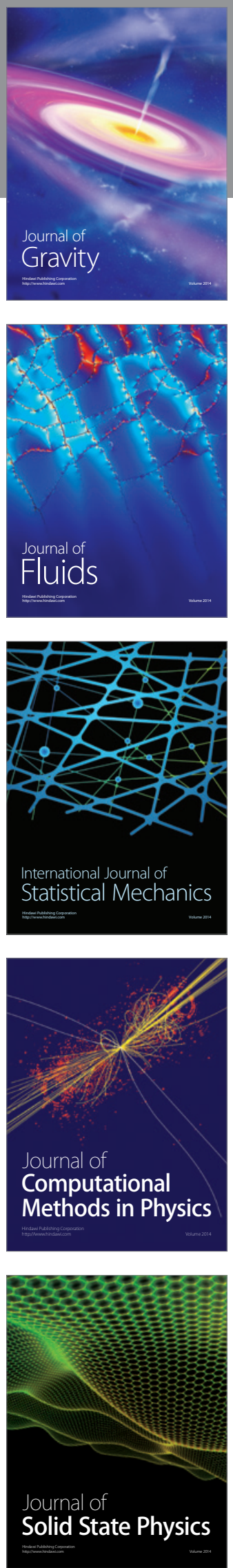

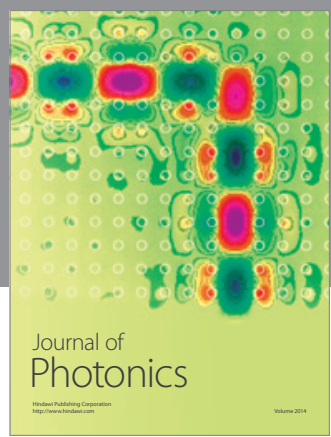

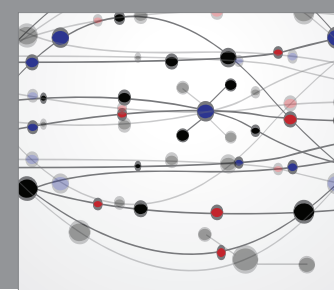

The Scientific World Journal

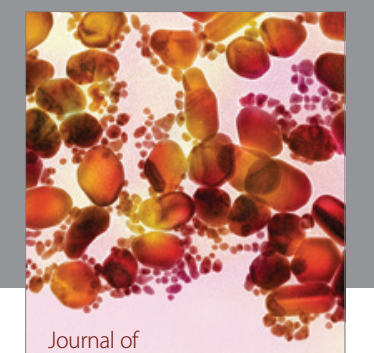

Soft Matter
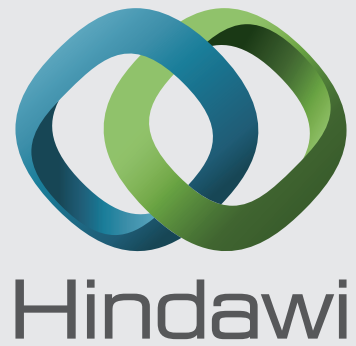

Submit your manuscripts at

http://www.hindawi.com
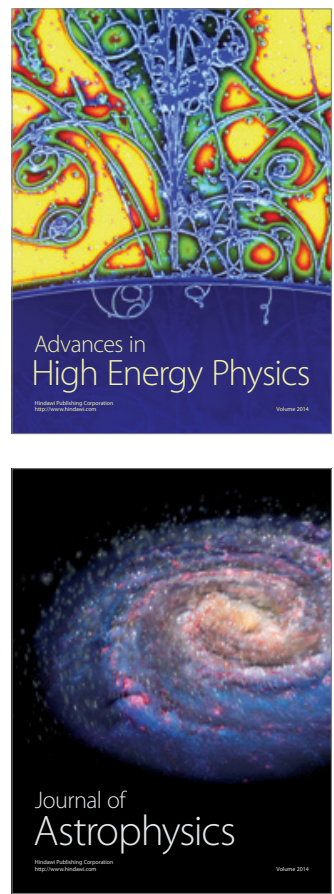
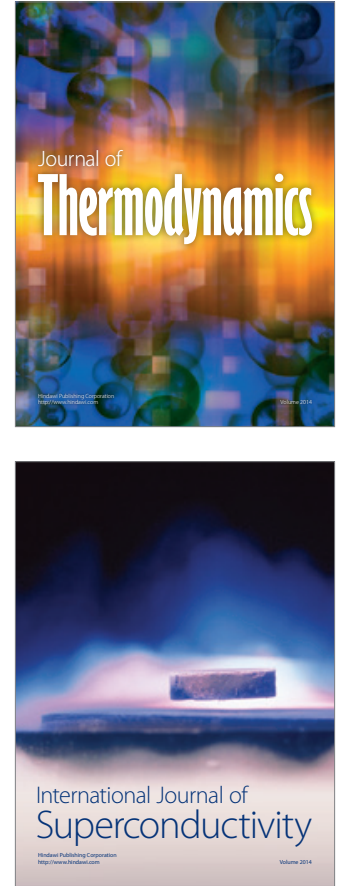
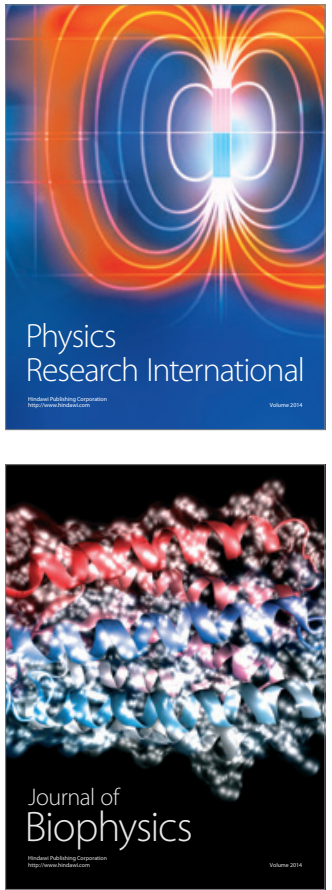
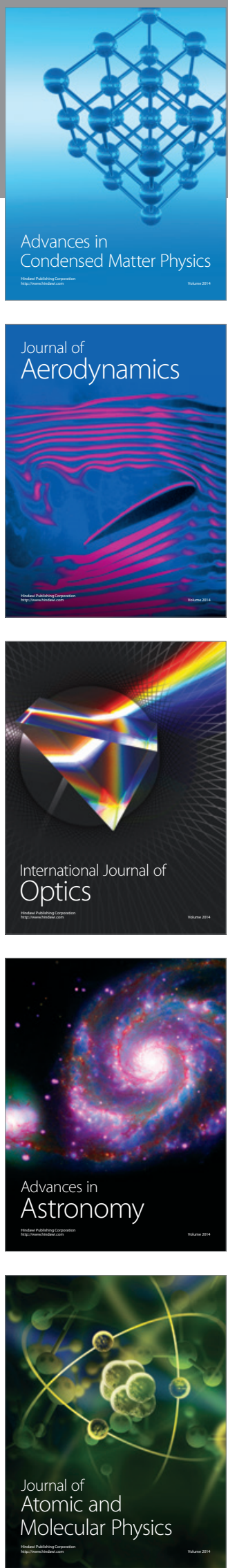